\title{
INHIBITION BY RECURRENT EXCITATION: A MECHANISM FOR SPIKE SYNCHRONIZATION IN A NETWORK OF COUPLED NEURONAL OSCILLATORS
}

\author{
By M. EGELHAAF* AND P. R. BENJAMIN \\ School of Biology, University of Sussex, Falmer, \\ Brighton, Sussex, BNI 9QG, U.K.
}

(Received 28 fuly 1981)

Two bilateral clusters of 2-7 peripheral-neurones ('Peripheral Bursters') are located on the left and right branches of the post-buccal nerve of the snail Lymnaea stagnalis quite separate from the ganglia of the CNS (Carriker, 1946; Egelhaaf, $198 \mathrm{I} a, b ; \mathrm{M}$. Egelhaaf \& P. R. Benjamin, in preparation). Isolation of single cells from these peripheral clusters shows that they are endogenously capable of bursting (Egelhaaf, $198 \mathrm{r} a$; M. Egelhaaf \& P. R. Benjamin, in preparation). In addition, the cells within one cluster as well as in heterolateral clusters are electrotonically coupled to one another, the coupling being stronger in the former case. Detailed evidence for this is given elsewhere (Egelhaaf, 1981 $a$; M. Egelhaaf $\& \mathrm{P}$. R. Benjamin, in preparation). This has allowed the investigation of the interactions between the endogenous oscillatory properties of single cells and network properties due to electrotonic coupling in a system which can easily be separated from the influence of other neurones (Egelhaaf, 1981 $a, b$; M. Egelhaaf \& P. R. Benjamin, in preparation). In this paper interactions among homolateral Peripheral Bursters will be discussed and it will be shown phenomenologically how spike synchrony can be established within bursts of cells which tend to fire asynchronously due to slightly different levels of excitability.

Standard intracellular recording techniques were used to monitor the activity of pairs of Peripheral Bursters on the same side of the buccal mass. They were separated from the group on the other side and the CNS by cutting branches of the postbuccal nerve (Fig. I $a$ ).

Homolateral Peripheral Bursters always show burst synchrony and often reveal synchronous I: I spike activity within bursts (Fig. I $b$ ). However, their electrotonical coupling is not strong enough to prevent them from occasionally having different levels of excitability. In this case firing at the beginning of a burst may not be synchronous although subsequently spike matching occurs. This is illustrated in Fig. I $c$. Cell $\mathrm{I}$ is initially less active than cell 2 and only later starts generating spikes, apparently in response to electrotonically mediated EPSPs arising from preceding spiking in cell 2. The firing frequency of the more active cell decreases following the delayed onset of firing in the less active cell. At this lower frequency both cells fire synchronously I: I. Hence, spiking in the less active cell leads to an inhibitory effect on the more active cell and, subsequently, to a matching of spike frequency in both cells.

- Present address: M.P.I. für Biologische Kybernetik, D-7400 Tübingen, Spemannstr. 38, F.R.G. 
(a)

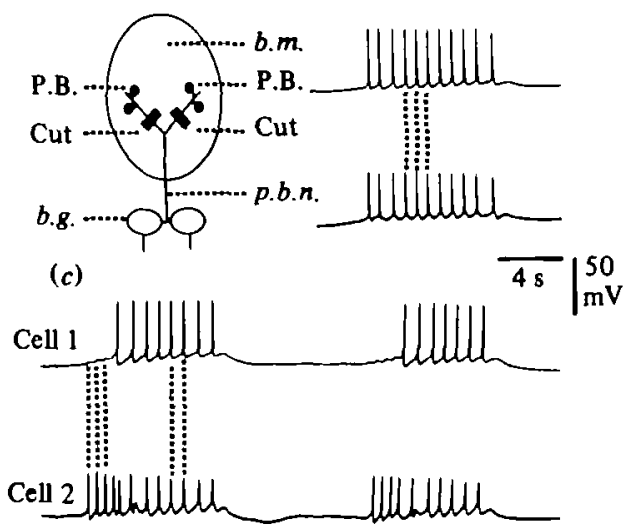

(d)

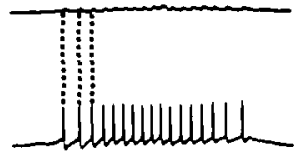

(e)

$\left.\overline{4 s}\right|_{\mathrm{mV}} ^{60}$

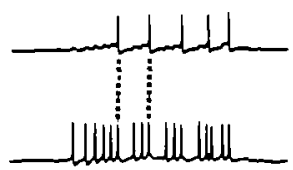

Fig. 1. Spike activity-dependent mutual influence among homolateral Peripheral Bursters. (a) Location of Peripheral Bursters on the buccal mass (b.m.). The post-buccal nerve (p.b.n.) has been cut, as indicated, to isolate Peripheral Bursters on one side of the buccal mass form those on the other side and the CNS (b.g.: buccal ganglia). (b) Synchronous spike activity within bursts of cells with approximately the same level of excitability. (c) Spike frequency matching in cells with different activity levels. Onset of spike activity in a less active cell (cell 1) leads to a decrease in spike frequency in a more active cell (cell 2) and to a subsequent matching of spike frequency in both cells. (Note the $1: 1$ EPSPs in cell 1 with spikes in cell 2 before it starts firing.) (d), (e) Dependency of the increase in interspike interval on spike activity in a coupled cell. (d) Cell in the upper trace hyperpolarized by intracellular hyperpolarizing current injection. (e) Cell in upper trace has been released from hyperpolarization sufficiently to allow the sporadic formation of spikes. The increase in interspike interval in the more active cell can be directly related to the generation of spikes in the coupled less active cell.

The manner in which spike activity in a less active Peripheral Burster affects the spike frequency in a more active one can be seen more clearly in Fig. Id, e. If one cell is sufficiently hyperpolarized to suppress spiking, EPSPs follow $\mathrm{I}: \mathrm{I}$ the spiking pattern of the coupled bursting cell (Fig. I $d$ ). The frequency of spikes within the burst does not show any discontinuities but gradually increases and then decreases. However, if the hyperpolarized cell is released from hyperpolarization sufficiently to allow the sporadic formation of spikes in response to an EPSP, each spike in the hyperpolarized cell is accompanied by a delay in the onset of the next spike in the other cell for some tenths of a second (Fig. I $e$ ). Hence, it appears that this increase in interspike interval in the more active cell is related to the occurrence of single spikes in the coupled, less active cell.

Nevertheless, spiking in the less active cell is - although necessary - not sufficient for the inhibitory phenomenon to occur. Instead, it will be shown next that the interspike interval is only prolonged, if the spike in the less active cell induces a regenerative response in the more active cell just after a spike generated by the usual rhythmic firing mechanism. Evidence for this is given in Fig. $2 a$, which shows two Peripheral Bursters spiking I I within a burst. Cell $\mathrm{I}$ is slightly more active than cell 2 , since spikes in cell 2 are always delayed in relation to the corresponding spikes in cell I (see dotted lines in Fig. $2 a$ ). This delay results in the cell 2 spike always evoking an electrotonic EPSP in cell $\mathrm{r}$. An increase in the interspike interval occurs only, if the initial depolarization is followed by a second depolarizing wave, which 


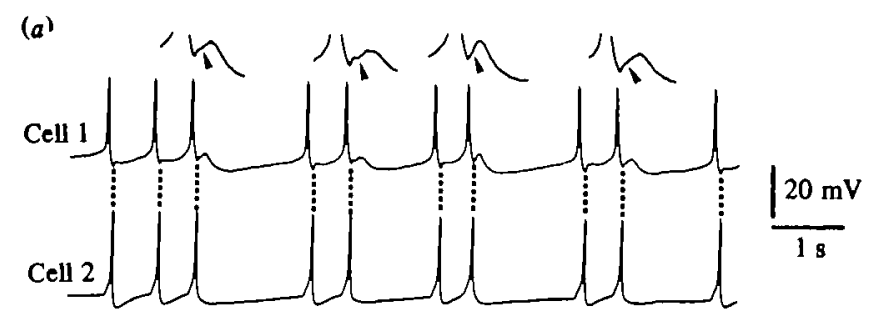

(b)

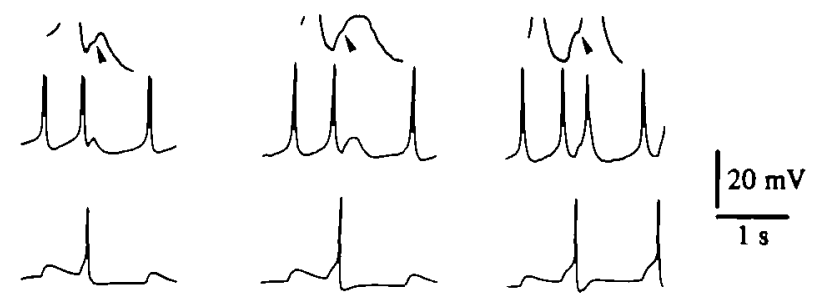

Fig. 2. The increase in interspike interval is due to an inactivation of the spike-generating mechanisms following extra spiking. (a) Delayed spikes in cell 2 lead to EPSP 8 in cell I (lines). An increase in interspike interval occurs only if a partial or complete regenerative response is induced by the EPSP, which can be distinguished from it by an inflexion in the overall depolarizing wave (see arrows in insets). (b) Evidence that the second phase of recurrent excitation is a blocked spike: its amplitude varies from EPSP-like depolarizations (left traces) to full-sized spikes (right traces).

in most cases can be clearly distinguished by an inflexion on the rising phase of the overall depolarizing waveform (see arrows in the insets of Fig. 2a). In the absence of this additional depolarizing wave, there is no delay in the timing of the next spike (Fig. 2a). These findings clearly demonstrate that the prolongation of the interspike interval is not directly mediated by spiking in the less active cell and, therefore, cannot be accounted for by mechanisms such as electrotonically transmitted post-spike hyperpolarization, since then it should have been induced by all cell 2 spikes in Fig. $2 a$. Instead, the increase in interspike interval appears to be directly related to the second depolarizing wave.

This second phase of the depolarizing wave caused by re-excitation is a regenerative response of the neurone rather than another EPSP. The evidence is as follows. First, the prolongation of the interspike interval induced by recurrent excitation occurs in preparations in which only two Peripheral Bursters are present. The second depolarizing phase occurs when only a single spike is recorded in the less active cell, so that the second phase cannot be a second EPSP. Secondly, since coupling among all homolateral cells is of approximately the same strength (Egelhaaf, 1981 $a$; Egelhaaf \& Benjamin, in preparation), the second phase of depolarization should be present in both cell $I$ and cell 2 , if it were an EPSP due to a spike in a third cell. However, Fig. 2(a) shows that it only occurs in cell I. Thirdly, the amplitude and shape of these additional depolarizations are highly variable, varying from EPSP-like depolarizations to full-sized spikes (Fig. $2 b$ ) in a manner that would not be expected from a second EPSP. These depolarizations thus appear to be recurrently induced regenerative responses, the amplitude of which depends 
on the state of refractoriness of the neurone. Hence, it can be concluded that the increase in interspike interval is due to these partial or complete regenerative responses, recurrently induced just after a spontaneously generated spike (Fig. 2a, b). Paradoxically, this means that recurrent excitation due to slightly asynchronous spiking in coupled cells can lead to inhibition and a decreased firing rate in the coupled neurones.

From the evidence presented here, it is suggested that spike synchronization in Lymnaea Peripheral Bursters with differing intrinsic activity levels can be accounted for in terms of an interaction of cellular and network properties, which can be called 'inhibition by recurrent excitation' (i.r.e.). The important endogenous cellular property is that a regenerative response of a cell, induced prematurely in relation to its projected spontaneous spike rhythm ('extra spike') delays the timing of the next spike to some extent, probably by resetting the spontaneous rhythmic firing mechanism. At present we are only able to account for i.r.e. phenomenologically and we have not attempted to explain it in terms of ionic mechanisms. The important network property consists of electrotonic coupling between the individual neurones within the network such that a spike in one produces an electrotonic EPSP and, depending on its timing in relation to the activity in the other cells, an extra spike. Hence, i.r.e. occurs in the case of initially asynchronously firing cells where feedback from each cell on to the others results in spike matching at the frequency of the least active neurone. I.r.e. probably does not occur if the entire cycle of membrane potential variation associated with burst and spike formation is in synchrony among the coupled neurones, as is the case when those neurones have the same endogenous activity levels.

Phenomenologically, this inhibition among homolateral Peripheral Bursters in Lymnaea is very similar to what has been called 'burst termination' in the electrotonically coupled trigger-group neurones (TGN) of Tritonia (Getting \& Willows, 1973, 1974) and subsequently in electrotonically coupled neurones in the visceral ganglion of Planorbis (Berry \& Pentreath, 1977; Pentreath \& Berry, 1977), in that prolongation of the interspike interval within spike trains depends critically on the firing of other cells within the network (e.g. compare Fig. $x d$, $e$ with Fig. 5 in Getting $\&$ Willows, 1974). (Note that this phenomenon, whether or not it is called 'burst termination', should not be confused with the termination of bursts in neurones, such as Peripheral Bursters, which show spontaneous endogenous membrane potential oscillations!) Getting \& Willows (1973, 1974) and later Berry \& Pentreath (1977; Pentreath \& Berry, 1977) suggest for their systems that the prolongation of the interspike interval and the concomitant accentuation of the post-spike hyperpolarization are the result of a reduction in junctional shunting, as the spikes in the coupled cells become more and more synchronized within a spike train. Thus their mechanism depends on spike synchrony, whereas evidence was given here that the prolongation in interspike interval in the Lymnaea Peripheral Bursters is due to i.r.e. and, therefore, based on a small but significant asynchrony of spikes in coupled cells. Hence, the mechanism proposed by Getting \& Willows (1973, 1974) for Tritonia cannot account for inhibition in the network of Peripheral Bursters in Lymnaea. 
An examination of the published data of Getting \& Willows from the TGNsystem of Tritonia suggests that i.r.e. may also play a role in 'burst termination' in this system, since in most of their recordings illustrating this phenomenon (Getting \& Willows, 1974, fig. I, figs. 5-7; Willows, 1976, fig. 2a) the increase in interspike interval is preceded either by spike doublets or other transient depolarizations which could be interpreted in terms of i.r.e. as extra spikes.

A number of examples are now available in which extra spiking induces prolongation of the following interspike interval (Calvin \& Schwindt, 1972; Calvin \& Sypert, 1975; Calvin \& Hartline, 1977) or of the interburst interval (Benjamin, 1978). Although the mechanisms which underlie extra spiking are apparently different among these examples, they have the feature in common with Peripheral Bursters that the extra spike-dependent prolongation of the interspike (interburst) interval is an intrinsic property of individual cells. However, the network of Peripheral Bursters differs in one respect from all these examples, in that the mechanism by which this extra spike is induced, i.e. electrotonically mediated recurrent excitation, is a property of the entire network.

This study was supported by a Studienstiftung des deutschen Volkes grant to M.E.

\section{REFERENCES}

Benjamin, P. R. (1978). Endogenous and synaptic factors affecting the bursting of double spiking molluscan neurosecretory neurones. In Abnormal Neuronal Discharges (ed. N. Chalazonitis and M. Boisson), pp. 205-216. New York: Raven Press.

BerRy, M. S. \& Pentreath, V. W. (1977). The integrative properties of electrotonic synapses. Comp. Biochem. Physiol. 57 A, 289-295.

Carriker, M. R. (1946). Morphology of the alimentary canal of the snail Lymnaea stagnalis apressa Say. Trans. Wisc. Acad. 38, $1-88$.

Calvin, W. H. \& Hartline, D. K. (1977). Retrograde invasion of lobster stretch receptor somata in control of firing rate and extra spike patterning. 7 . Neurophysiol. 40, 106-1 18.

CAlvin, W. H. \& Schwindt, P. C. (1972). Steps in production of motoneuron spiles during rhythmic firing. F. Neurophysiol. 35, 297-301.

Calvin, W. H. \& SYPert, P. O. (I 975). Cerebral cortex neurons with extra spikes: a normal substrate for epileptic discharges? Brain Res. 83, 498-503.

Egelhaf, M. ( $198 \mathrm{I} a$ ). Coupled neuronal oscillators in the snail Lymnaea stagnalis: endogenous cellular properties and network interactions. Diplom thesis, Universitat Tubingen, Federal Republic of Germany.

EgelhaAf, M. (ig8 I $b$ ). Network interactions of electrotonically coupled neuronal oscillators. Verh. Dtsch. Zool. Ges. (In the Press.)

Getting, P. A. \& Willows, A. O. D. (1973). Burst formation in electrically coupled neurons. Brain Res. 63, 424-429.

Getting, P. A. \& Willows, A. O. D. (1974). Modification of neuron properties by electrotonic synapses. II. Burst formation by electrotonic synapses. F. Neurophysiol. 37, 858-868.

Pentreath, V. W. \& Berry, M. S. (1977). Electrotonic synapses in the visceral ganglion of Planorbis. Experientia 33, 354-355.

Willows, A. O. D. (1976). Triggerneurons in the mollusk Tritonia. In Newral Control of Lacomotion (ed. R. D. Herman, S. Grillner, P. S. G. Stein and D. G. Stuart), pp. 327-349. New York: Plenum Press. 